\title{
Fuzzy strongly pre-irresolute functions
}

\author{
N. Gowrisankar, N. Rajesh \\ (Presented by V. V. Sharko)
}

\begin{abstract}
In this paper, we have introduced and studied a new class of fuzzy irresolute functions, called fuzzy strongly pre-irresolute functions by using fuzzy preopen sets.
\end{abstract}

2000 MSC. 54A40.

Key words and phrases. Fuzzy topology, fuzzy preopen sets, fuzzy pre-irresolute functions.

\section{Introduction}

The concept of fuzzy set was introduced by Zadeh [9]. Based on the concept of fuzzy sets, Chang [2] introduced and developed the concept of fuzzy topological spaces. Since then various important notions in the classical topology such as continuous functions [2] have been extended to fuzzy topological spaces. Fuzzy irresolutness is one of the main topics in fuzzy topology. In this paper, we have introduced and studied a new class of fuzzy irresolute functions, called fuzzy strongly pre-irresolute functions by using fuzzy preopen sets. This concept can be extended to Intuitionstic fuzzy topology and Ideal fuzzy topology.

\section{Preliminaries}

Now, we recall some basic notions and results that are used in the sequel.

Definition 2.1. A fuzzy topology on a nonempty set $X$ is a family $\tau$ of fuzzy subsets of $X$ which satisfies the following three conditions:

(i) $0,1 \in \tau$,

(ii) If $g, h \in \delta$, their $g \wedge h \in \tau$,

Received 27.10.2008 
(iii) If $f_{i} \in \tau$ for each $i \in I$, then $\vee_{i \in I} f_{i} \in \tau$.

The pair $(X, \tau)$ is called a fuzzy topological space [2].

Definition 2.2. Members of $\tau$ are called fuzzy open sets [2] and the complement of fuzzy open sets are called fuzzy closed sets [2], where the complement of a fuzzy set $A$, denoted by $A^{c}$, is $1-A$.

Definition $2.3([7])$. The fuzzy subset $x_{a}$ of a non-empty set $X$, with $x \in X$ and $0<a \leq 1$ defined by

$$
x_{a}(p)= \begin{cases}a & \text { if } p=x \\ 0 & \text { if } p \neq x\end{cases}
$$

is called a fuzzy point in $X$ with suppost $x$ and value $a$. The fuzzy point $x_{1}$ is called crisp point.

Definition 2.4 ([7]). Let $\lambda$ be fuzzy set in $X$ and $x_{a}$ a fuzzy point in $X$. We say that $x_{a} \in \lambda$ if and only if $x_{a} \leq \lambda$.

Definition 2.5. A fuzzy set B is a quasi neighbourhood [7] (q-neighbourhood, for short) of $A$ if and only if there exists a fuzzy open set $U$ such that $A q U \leq B$.

Definition 2.6. A fuzzy set $\lambda$ is a pre-q-neighbourhood (for short, pre$q-n b d)$ of $\mu$ if and only if there exists a fuzzy preopen set $\gamma$ such that $\lambda q \gamma$ and $\gamma \leq \mu$.

Definition 2.7. Let $\lambda$ be any fuzzy set in the fuzzy topological space $(X, \tau)$. Then we define the fuzzy preinterior [8] of $\lambda=p \operatorname{Int}(\lambda)=\vee\{\mu: \mu$ is fuzzy preopen and $\mu \leq \lambda\}$ and the fuzzy preclosure of $\lambda=p \operatorname{Cl}(\lambda)=$ $\wedge\{\mu: \mu$ is fuzzy preclosed and $\mu \leq \lambda\}$.

Remark 2.1. For any fuzzy $\lambda$ in a fuzzy topological space $(X, \tau), 1-$ $p \mathrm{Cl}(\lambda)=p \operatorname{Int}(1-\lambda)$ and $p \operatorname{Cl}(\lambda)=1-p \operatorname{Int}(\lambda)$.

Definition 2.8 ([2]). Let $X$ and $Y$ be two fuzzy topological spaces. Let $A \in I^{X}, B \in I^{Y}$. Then $f(A)$ is a fuzzy subset of $Y$, defined by $f(A): Y \rightarrow[0,1]$

$$
f(A)(y)= \begin{cases}\sup _{x \in f^{-1}(\{y\})} A(x) & \text { if } f^{-1}(\{y\}) \neq \varnothing \\ 0 & \text { if } f^{-1}(\{y\})=\varnothing\end{cases}
$$

and $f^{-1}(B)$ is a fuzzy subset of $X$, defined by $f^{-1}(B)(x)=B(f(x))$. 
Lemma 2.1 ([1]). Let $f: X \rightarrow Y$ be a function and $\lambda_{\alpha} \alpha \in I$ be a family of fuzzy sets of $Y$, then

(i) $f^{-1}\left(\cup \lambda_{\alpha}\right)=\cup f^{-1}\left(\lambda_{\alpha}\right)$ and

(ii) $f^{-1}\left(\cap \lambda_{\alpha}\right)=\cap f^{-1}\left(\lambda_{\alpha}\right)$.

Lemma 2.2 ([1]). For functions $f_{i}: X_{i} \rightarrow Y_{i}$ and fuzzy sets $\lambda_{i}$ of $Y_{i}, i=1,2$, we have $\left(f_{1} \times f_{2}\right)^{-1}\left(\lambda_{1} \times \lambda_{2}\right)=f_{1}^{-1}\left(\lambda_{1}\right) \times f_{2}^{-1}\left(\lambda_{2}\right)$.

Lemma 2.3 ([1]). Let $g: X \rightarrow X \times Y$ be the graph of a function $f: X \rightarrow Y$. Then, if $\lambda$ is a fuzzy set of $X$ and $\mu$ is a fuzzy set of $Y$, $g^{-1}(\lambda \times \mu)=\lambda \wedge f^{-1}(\mu)$.

Definition 2.9. A fuzzy singleton $x_{\alpha}$ is said to be fuzzy pre- $\theta$-cluster [5] point of a fuzzy subset $A$ of $X$ if the fuzzy preclosure if every fuzzy pre$q$-nbd of $x_{\alpha}$ is q-coincident with $A$. The union of all fuzzy pre- $\theta$-cluster points of $A$ is called the pre- $\theta$-closure [5] of $A$ and is denoted by $p \mathrm{Cl}_{\theta}(A)$. $A$ fuzzy set $A$ of $X$ is called fuzzy pre- $\theta$-closed [5] if $A=p \mathrm{Cl}_{\theta}(A)$, and the complement of a fuzzy pre- $\theta$-closed set is called fuzzy pre- $\theta$-open [5]. The union of all fuzzy pre- $\theta$-open sets of $X$ contained in $A$ is called the fuzzy pre- $\theta$-interior of $A$ and is denoted by $p \operatorname{Int}_{\theta}(A)$.

Remark 2.2. For a fuzzy set $A$ of $X, A \leq p \mathrm{Cl}(A) \leq p \mathrm{Cl}_{\theta}(A)$ and hence each fuzzy pre- $\theta$-closed set is fuzzy preclosed.

Lemma 2.4. For a fuzzy set $A$ of $X$, we have that

(i) $p \operatorname{Int}_{\theta}(1-A)=1-p \mathrm{Cl}_{\theta}(A)$;

(ii) $p \mathrm{Cl}_{\theta}(1-A)=1-p \operatorname{Int}_{\theta}(A)$.

Definition 2.10. A function $f: X \rightarrow Y$ is said to be fuzzy pre-irresolute [3] if $f^{-1}(V) \in F P O(X)$ for each $V \in F P O(Y)$.

Definition 2.11. A function $f: X \rightarrow Y$ is said to be fuzzy weakly preirresolute [6] if for any fuzzy singleton $x_{\alpha}$ in $X$ and each fuzzy preopen set $V$ of $Y$ containing $f\left(x_{\alpha}\right)$, there exists a fuzzy preopen set $U$ containing $x_{\alpha}$ such that $f(U) \leq p \mathrm{Cl}(V)$.

\section{Fuzzy strongly pre-irresolute functions}

Definition 3.1. A function $f: X \rightarrow Y$ is said to be fuzzy strongly preirresolute if for any fuzzy singleton $x_{\alpha}$ in $X$ and each fuzzy preopen set $V$ containing $f\left(x_{\alpha}\right)$, there exists a fuzzy preopen set $U$ containing $x_{\alpha}$ such that $f(p \mathrm{Cl}(U)) \leq V$. 
It is clear that, every fuzzy strongly pre-irresolute function is fuzzy pre-irresolute. But the converse is not true in general as it can be seen from the following example.

Example 3.1. Let $(X, \tau)$ as in Example 2.7 of [4] and $\tau=\{0,1\}$. Define a function $f: X \rightarrow Y$ by $f(x)=y$ and $f(y)=x$. Then $f$ is fuzzy preirresolute but not fuzzy strongly pre-irresolute.

Theorem 3.1. For a function $f: X \rightarrow Y$, the following properties are equivalent:

(i) $f$ is fuzzy strongly pre-irresolute;

(ii) for each fuzzy singleton $x_{\alpha}$ of $X$ and each fuzzy preopen set $V$ of $Y$ containing $f\left(x_{\alpha}\right)$, there exists a fuzzy regular preopen set $U$ of $X$ containing $x_{\alpha}$ such that $f\left(p \mathrm{Cl}_{\theta}(U)\right) \leq V$;

(iii) for each fuzzy singleton $x_{\alpha}$ of $X$ and each fuzzy preopen set $V$ of $Y$ containing $f\left(x_{\alpha}\right)$, there exists a fuzzy regular preopen set $U$ in $X$ containing $x_{\alpha}$ such that $f(U) \leq U$;

(iv) for each fuzzy singleton $x_{\alpha}$ of $X$ and each fuzzy preopen set $V$ of $Y$ containing $f\left(x_{\alpha}\right)$, there exists a fuzzy pre- $\theta$-open set $U$ in $X$ containing $x_{\alpha}$ such that $f(U) \leq V$;

(v) $f^{-1}(V)$ is fuzzy pre- $\theta$-open in $X$ for every $V \in F P O(Y)$;

(vi) $f^{-1}(F)$ is fuzzy pre- $\theta$-closed in $X$ for every $F \in F P C(Y)$;

(vii) $f\left(p \mathrm{Cl}_{\theta}(A)\right) \leq p \mathrm{Cl}(f(A))$ for every fuzzy subset $A$ of $X$;

(viii) $p \mathrm{Cl}_{\theta}\left(f^{-1}(B)\right) \leq f^{-1}(p \mathrm{Cl}(B))$ for every fuzzy subset $B$ of $Y$.

Proof. The implications $(i) \Rightarrow(i i) \Rightarrow(i i i) \Rightarrow(i v) \Rightarrow(i)$ are obivious.

$(i v) \Rightarrow(v)$ : Let $V \in F P O(Y)$. Suppose that $x_{\alpha} \in f^{-1}(V)$. Therefore, $f(x) \in V$ and then there exists a fuzzy pre- $\theta$-open set $U$ in $X$ containing $x_{\alpha}$ such that $f(U) \leq V$. Therefore, we have $x_{\alpha} \in U \leq f^{-1}(V)$. Since the union of fuzzy pre- $\theta$-open sets is fuzzy pre- $\theta$-open, $f^{-1}(V)$ is fuzzy pre- $\theta$-open in $X$.

$(v) \Rightarrow(v i)$ : This is obvious.

$(v i) \Rightarrow(v i i)$ : Let $B$ be any fuzzy subset of $Y$. Since $p \operatorname{Cl}(A)$ is fuzzy preclosed in $Y$, by $(v i), f^{-1}(p \mathrm{Cl}(f(A)))$ is fuzzy pre- $\theta$-closed in $X$ and we have

$$
\begin{aligned}
p \mathrm{Cl}_{\theta}(A) \leq p \mathrm{Cl}_{\theta}\left(f^{-1}(\right. & f(A))) \\
& \leq p \mathrm{Cl}_{\theta}\left(f^{-1}(p \mathrm{Cl}(f(A)))\right)=f^{-1}(p \mathrm{Cl}(f(A))) .
\end{aligned}
$$


Therefore, we obtain $f\left(p \mathrm{Cl}_{\theta}(A)\right) \leq p \mathrm{Cl}(f(A))$.

$(v i i) \Rightarrow(v i i i)$ : Let $B$ be any fuzzy subset of $Y$. By (vii), we obtain

$$
f\left(p \mathrm{Cl}_{\theta}\left(f^{-1}(B)\right)\right) \leq p \operatorname{Cl}\left(f\left(f^{-1}(B)\right)\right) \leq p \operatorname{Cl}(B)
$$

and hence

$$
p \mathrm{Cl}_{\theta}\left(f^{-1}(B)\right) \leq f^{-1}(p \mathrm{Cl}(B)) .
$$

(viii) $\Rightarrow(i)$ : Let $x_{\alpha}$ be a fuzzy singleton of $X$ and $V$ be a fuzzy preopen set of $Y$ containing $f\left(x_{\alpha}\right)$. Since $1-V \in F P C(Y)$, we have

$$
p \mathrm{Cl}_{\theta}\left(f^{-1}(1-V)\right) \leq f^{-1}(p \mathrm{Cl}(1-V))=f^{-1}(1-V) .
$$

Therefore, $f^{-1}(1-V)$ is fuzzy pre- $\theta$-closed in $X$ and $f^{-1}(V)$ is a fuzzy pre- $\theta$-open set containing $x_{\alpha}$. Then there exists a fuzzy preopen set $U$ of $X$ containing $x_{\alpha}$ such that $p \mathrm{Cl}(U) \leq f^{-1}(V)$; hence $f(p \mathrm{Cl}(U)) \leq V$. This shows that $f$ is fuzzy strongly pre-irresolute.

Definition 3.2. A fuzzy space $(X, \tau)$ is said to be fuzzy pre-regular if for each fuzzy singleton $x_{\alpha}$ and each fuzzy preopen set $A$ there exists a fuzzy preopen set $B$ such that $x_{\alpha} \in B \leq p \operatorname{Cl}(B) \leq A$.

Theorem 3.2. A fuzzy pre-irresolute function $f: X \rightarrow Y$ is fuzzy strongly pre-irresolute if and only if $X$ is fuzzy pre-regular.

Proof. Necessity: Let $f: X \rightarrow Y$ be the identity function. Then $f$ is fuzzy pre-irresolute and fuzzy strongly fuzzy pre-irresolute by the hypothesis. For any $U \in F P O(X)$ and any fuzzy singleton $x_{\alpha}$ of $U$, we have $f\left(x_{\alpha}\right)=x_{\alpha} \in U$ and there exists a fuzzy preopen set $G$ of $X$ containing $x_{\alpha}$ such that $f(p \mathrm{Cl}(G)) \leq U$. Therefore, we have $x \in G \leq p \mathrm{Cl}(G) \leq U$. By definition, $X$ is fuzzy pre-regular.

Corollary 3.1. Let $X$ be a fuzzy pre-regular space. Then $f: X \rightarrow Y$ is fuzzy strongly pre-irresolute if and only if $f$ is fuzzy pre-irresolute.

Proof. This follows immediately from Theorem 3.2.

Theorem 3.3. If $f: X \rightarrow Y$ is fuzzy weakly pre-irresolute and $Y$ is fuzzy pre-regular, then $f$ is fuzzy strongly pre-irresolute.

Proof. Let $x_{\alpha}$ be a fuzzy singleton of $X$ and let $V$ be a fuzzy preopen set containing $f\left(x_{\alpha}\right)$. Then there exists a fuzzy preopen set $G$ containing $f\left(x_{\alpha}\right)$ such that $f\left(x_{\alpha}\right) \in G \leq p \mathrm{Cl}(G) \leq V$. By Theorem 3.1, there exists a fuzzy preopen set $U$ containing $x_{\alpha}$ such that $f(p \mathrm{Cl}(U)) \leq p \mathrm{Cl}(G)$. Thus, $f(p \mathrm{Cl}(G)) \leq V$ and $f$ is fuzzy strongly pre-irresolute. 
Corollary 3.2. If $f: X \rightarrow Y$ is a function and $Y$ is fuzzy pre-regular, then the following statements are equivalent:

(i) $f$ is fuzzy strongly pre-irresolute;

(ii) $f$ is fuzzy irresolute;

(iii) $f$ is fuzzy weakly pre-irresolute.

Theorem 3.4. Let $f: X \rightarrow Y$ be a function, where $X$ is product related to $Y$, and $g: X \rightarrow X \times Y$, the graph function of $g$. If $g$ is fuzzy strongly pre-irresolute, then $f$ is so and $X$ is fuzzy pre-regular.

Proof. Suppose that $g$ is fuzzy strongly pre-irresolute. First, we show that $f$ is fuzzy strongly pre-irresolute. Let $x_{\alpha}$ be a fuzzy singleton of $X$ and $V$ be a fuzzy preopen set of $Y$ containing $f\left(x_{\alpha}\right)$. Then $1 \times V$ is a fuzzy preopen set of $X \times Y$ containing $g\left(x_{\alpha}\right)$. Since $g$ is fuzzy strongly pre-irresolute, there exists a fuzzy preopen set $U$ of $X$ containing $x_{\alpha}$ such that $g(p \mathrm{Cl}(U)) \leq 1 \times V$. Therefore, we obtain $f(p \mathrm{Cl}(U)) \leq V$. This shows that $f$ is fuzzy strongly pre-irresolute. Next, we show that $X$ is fuzzy pre-regular. Let $U$ be a fuzzy preopen set of $X$ containing $x_{\alpha}$. Since $g(x) \in U \times 1$ and $U \times 1$ is fuzzy preopen in $X \times Y$, there exists a fuzzy preopen set $G$ of $X$ containing $x_{\alpha}$ such that $g(p \mathrm{Cl}(G))<U \times 1$. Therefore, we obtain $x_{\alpha} \in G \leq p \mathrm{Cl}(G) \leq U$ and hence $X$ is fuzzy pre-regular.

Definition 3.3. A function $f: X \rightarrow Y$ is said to be fuzzy strongly preopen if $f(U) \in F P O(Y)$ for each $U \in F P O(X)$.

Lemma 3.1. If $f: X \rightarrow Y$ is fuzzy pre-irresolute and $V$ is fuzzy pre- $\theta$ open in $Y$, then $f^{-1}(V)$ is fuzzy pre- $\theta$-open in $X$.

Proof. Let $V$ be a fuzzy pre- $\theta$-open set of $Y$ and $x_{\alpha} \in f^{-1}(V)$. Then there exists $W \in F P O(Y)$ such that $f\left(x_{\alpha}\right) \in W \leq p \mathrm{Cl}(W) \leq V$. Since $f$ is fuzzy pre-irresolute, we have $f^{-1}(W) \in F P O(X)$ and $p \mathrm{Cl}\left(f^{-1}(W)\right) \leq$ $f^{-1}(p \mathrm{Cl}(W))$. Therefore, we have $x_{\alpha} \in f^{-1}(W) \leq p \mathrm{Cl}\left(f^{-1}(W)\right) \leq$ $f^{-1}(V)$ and $f^{-1}(V)$ is fuzzy pre- $\theta$-open in $X$.

Theorem 3.5. Let $f: X \rightarrow Y$ and $g: Y \rightarrow Z$ be functions. Then the following properties hold:

(i) If $f$ is fuzzy strongly pre-irresolute and $g$ is fuzzy pre-irresolute, then $g \circ f: X \rightarrow Z$ is fuzzy strongly pre-irresolute;

(ii) If $f$ is fuzzy pre-irresolute and $g$ is fuzzy strongly pre-irresolute, then $g \circ f: X \rightarrow Z$ is fuzzy strongly pre-irresolute; 
(iii) If $f$ is fuzzy strongly pre-irresolute and $g$ is fuzzy weakly pre-irresolute, then $g \circ f: X \rightarrow Z$ is fuzzy weakly pre-irresolute;

(iv) If $f$ is fuzzy strongly preopen bijection and $g \circ f: X \rightarrow Z$ is fuzzy strongly pre-irresolute, then $g$ is fuzzy strongly pre-irresolute.

Proof. $(i)$ : This is obvious.

(ii): This follows immediately from Theorem 3.1 and Lemma 3.1.

(iii): Let $W \in F P O(Z)$. Since $g \circ f$ is fuzzy strongly pre-irresolute, $(g \circ f)^{-1}(W)$ is fuzzy pre- $\theta$-open in $X$. Since $f$ is fuzzy strongly preopen and bijective, $f^{-1}$ is fuzzy pre-irresolute and by Lemma 3.1, we have $g^{-1}(W)=f\left((g \circ f)^{-1}(W)\right)$ us fuzzy pre- $\theta$-open in $Y$. Therefore,Hence by Theorem 3.1, $g$ is fuzzy strongly pre-irresolute.

$(i v)$ : Let $x_{\alpha}$ be a fuzzy singleton of $X$ and $y_{\beta}=f\left(x_{\alpha}\right)$. Let $V$ be a fuzzy regular preopen in $Z$ containing $g\left(y_{\beta}\right)=g\left(f\left(x_{\alpha}\right)\right)$. Since $g$ is fuzzy weakly pre-irresolute, there exists a fuzzy regular preopen set $U$ containing $y_{\beta}$ such that $f(p \mathrm{Cl}(G)) \leq U$. Hence

$$
(g \circ f)(p \mathrm{Cl}(G))=g(f(p \mathrm{Cl}(G))) \leq g(U) \leq V .
$$

Therefore, $g \circ f$ is fuzzy weakly pre-irresolute.

\section{References}

[1] K. K. Azad, On fuzzy semicontinuity, fuzzy almost continuity and fuzzy weakly continuity // J. Math. Anal. Appl., 82 (1981), 297-305.

[2] C. L. Chang, Fuzzy topological spaces // J. Math. Anal. Appl., 24 (1968), 182-190.

[3] M. A. Fath Alla, On fuzzy topological spaces, Ph. D Thesis, Assuit University (Egypt), 1984.

[4] M. Ganstar, D. M. Georgion, S. Jafari and S. P. Moshokoa, On some applications of fuzzy points // Appl. Gen. Topology, 6(2) (2005), 119-133.

[5] N. Gowrisankar and N. Rajesh, Fuzzy regular preopen sets, (submitted).

[6] N. Gowrisankar and N. Rajesh, Fuzzy weakly pre-irresolute functions, (submitted).

[7] P. Pao-Ming and L. Ying-Ming, Fuzzy topology I Neighbourhood structure of a fuzzy point and Moore-Smith Convergence // J. Math. Anal. Appl., 76 (1980), $571-599$.

[8] M. K. Singal and N. Prakash, Fuzzy preopen sets and fuzzy preseparation axioms // Fuzzy sets and Systems, 44 (1991), 273-281.

[9] L. A. Zadeh, Fuzzy sets // Information and Control, 8 (1965), 338-353. 
Contact information

N. Gowrisankar

249, Second Floor,

S. G. Palaiya,

C. V. Raman Nagar

Bangalore 560093,

India

N. Rajesh

Department of Mathematics

Rajah Serfoji Govt. College

Thanjavur-613005

Tamilnadu,

India

E-Mail: nrajesh_topology@yahoo.co.in 